\title{
Multifocal intraosseous calvarial hemangioma misdiagnosed as subgaleal lipoma
}

\author{
Joo-Hak Kim ${ }^{1}$, \\ Chang Hwan Ahn', \\ Kyung-Hee Kim ${ }^{3}$, \\ Sang-Ha $\mathrm{Oh}^{1,2}$ \\ ${ }^{1}$ Department of Plastic and \\ Reconstructive Surgery, ${ }^{2}$ Brain Research \\ Institute, and ${ }^{3}$ Department of \\ Pathology, School of Medicine, \\ Chungnam National University, \\ Daejeon, Korea
}

\begin{abstract}
Intraosseous hemangioma is a rare, slow-growing, benign tumor of blood vessels. Primary hemangioma of the skull is a benign lesion that may appear as a palpable mass or accidentally detected during image evaluation. Simple radiography is the most commonly used technique to localize a lesion and computed tomography (CT) may help determine the effect of a lesion. We report a case of multifocal intraosseous calvarial hemangioma developed in the subgaleal plane of an elderly male patient. Ultrasonography examination revealed hyperechoic striated septae parallel to the skin and discontinuity of the focal cortex, however, the underlying bone cortex appeared relatively intact. No significant flow is observed on Doppler ultrasonography. Based on these evaluations, the mass was interpreted by a radiologist as a subgaleal lipoma. This case highlights the importance of additional CT examination in a patient presenting with a scalloping sign of the underlying calvarium. Clinicians also should be aware of the possibility of intraosseous calvarial hemangiomas in lesion. Furthermore, the proper choice of congenital vascular malformation term is still quite confusing with misconception present in the literature.
\end{abstract}

Keywords: Lipoma / Skull / Ultrasonography / Vascular malformations

\section{INTRODUCTION}

Ultrasonography is a useful noninvasive method to detect the exact location of subcutaneous tumors before surgery. However, findings are not always in accordance with the actual echogenicity of the soft tissue mass. Importantly, there is a possibility of misdiagnosis atypical cases when using ultrasonography.

Intraosseous hemangioma is a rare, slow-growing, benign tumor of blood vessels accounting for $0.7 \%$ of all neoplasms of bone [1]. Meanwhile, calvarial hemangiomas, which account for $0.2 \%$ of all bone neoplasms, are more than three times more common in women than men. Although calvarial hemangiomas can be seen at any age, patients are usually treated between

\section{Correspondence: Sang-Ha Oh}

Department of Plastic and Reconstructive Surgery, School of Medicine, Chungnam

National University, 282 Munhwa-ro, Jung-gu, Daejeon 35015, Korea

E-mail: djplastic@cnu.ac.kr
20 and 40 years of age [2]. Intraosseous hemangiomas are most commonly found in the vertebral column and rarely seen in the calvarium. Among the calvarial bones, the parietal bone is the most commonly involved; frontal, occipital and temporal bones are less frequently involved [3]. The intraosseous calvarial hemangiomas may be monofocal or multifocal, but the majority of reported cases are monofocal. In very rare cases, multifocal hemangiomas may occur [4].

\section{CASE REPORT}

\section{History and presentation}

A 60-year-old male patient presented with a complaint of a painless palpable mass in the left forehead region that had been slowly enlarging for past 2 years. There was no history of forehead trauma. Medical and surgical histories were insignificant. 


\section{Physical examination}

On physical examination, swelling (diameter, $2.5 \mathrm{~cm}$; height, 7 $\mathrm{mm}$ ) was observed without change of the overlying skin in the left forehead area (Fig. 1A). The swelling was not so firm, nonfluctuant, non-tender and slightly movable without any bruit. There was no change in size with compression. The head shape was symmetric. There were no other abnormal findings upon general and neurologic examinations.

\section{Radiologic imaging}

Ultrasonography revealed a well-defined margin adjacent to the left frontal bone in the subgaleal layer, with a hypoechoic lesion $(2.2 \mathrm{~cm} \times 0.3 \mathrm{~cm})$. Hyperechoic inside septation and focal cortical discontinuity were also observed, while the underlying bone cortex appeared relatively intact. No significant flow is observed on Doppler sonography, suggesting no evidence of internal vascularity (Fig. 1B). Thus, the mass was interpreted as a subgaleal lipoma by a radiologist.

\section{Excisional biopsy}

Complete excision was conducted under local anesthesia. The left frontal lesion was well delineated at subgaleal layer and the mass was reddish brown in color and appeared to originate from bony cortex. This lesion was attached to the outer cortex of the frontal bone and revealed hemorrhagic features with a cluster of small vessels. After excision of the mass, the cortical bone was relatively intact, but there was erosion of the outer table of the skull (Fig. 2A). To prevent possible bone bleeding, careful hemostasis with electrocauterization was performed. The mass of left frontal lesion was soft, well delineated, reddish
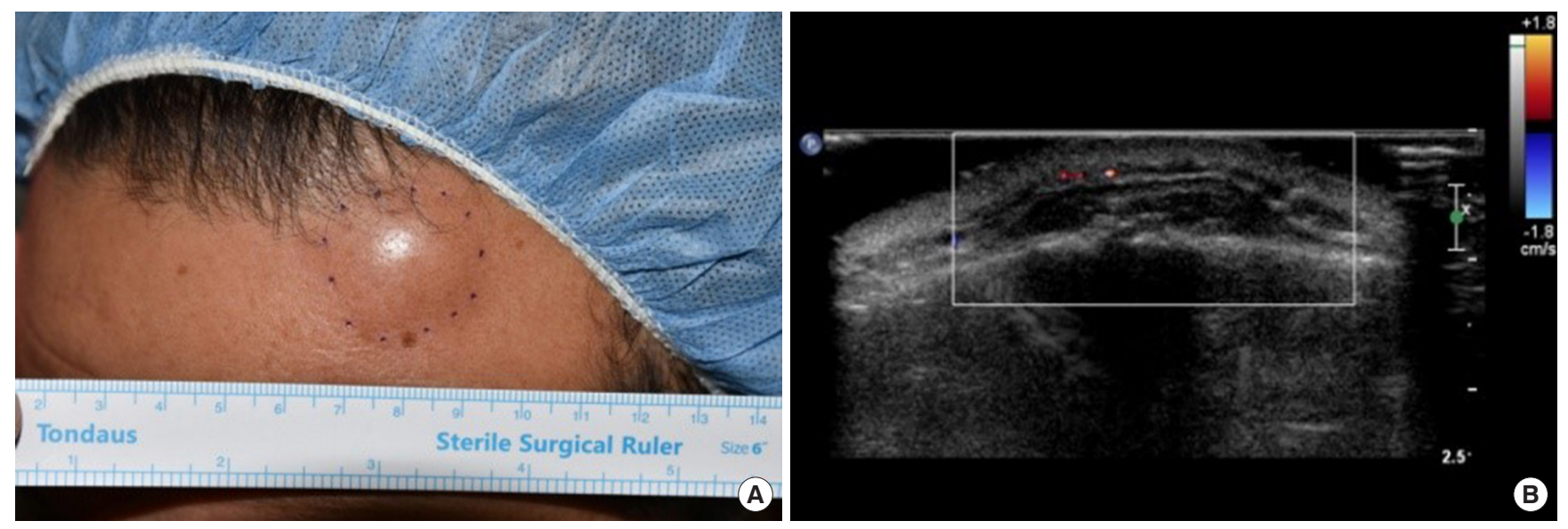

Fig. 1. Preoperative photo and ultrasonographic findings. (A) A swelling (diameter, $2.5 \mathrm{~cm}$; height, $7 \mathrm{~mm}$ ) was observed without change of the overlying skin in the left forehead area. (B) Ultrasonography revealed a well-defined margin adjacent to the left frontal bone in the subgaleal layer, with a hypoechoic lesion $2.2 \mathrm{~cm} \times 0.3 \mathrm{~cm}$ in size. It revealed hyperechoic striated septae parallel to the skin suggestive of lipoma. No significant flow is observed on Doppler sonography, which indicated no evidence of internal vascularity.
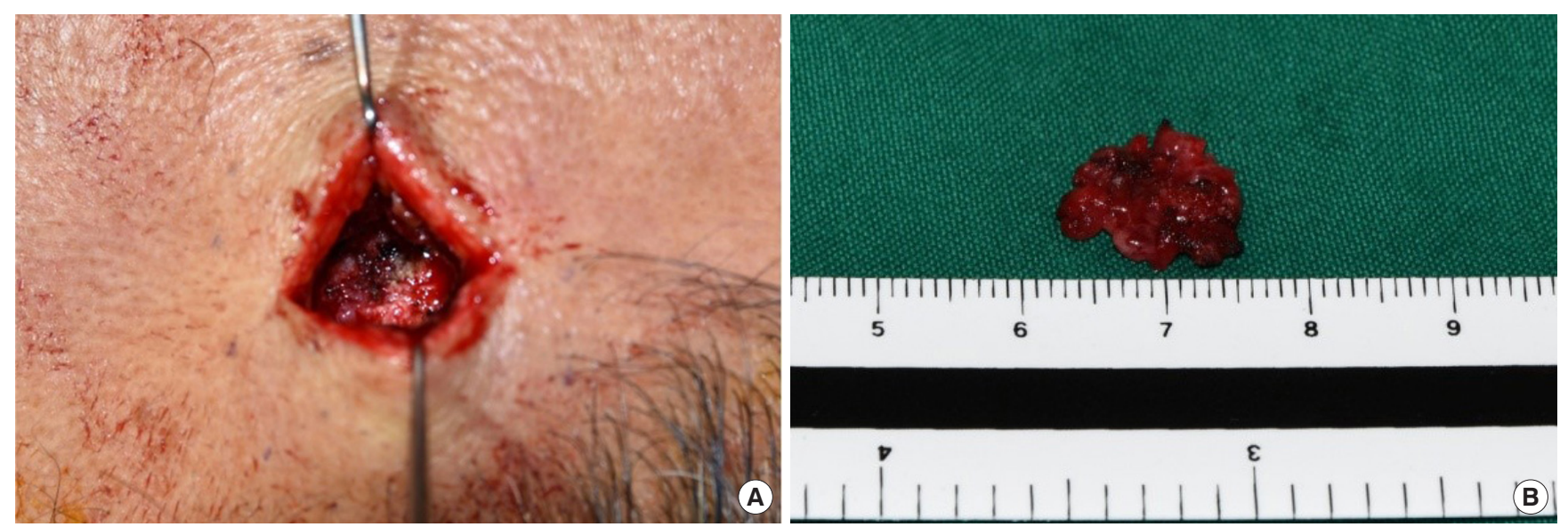

Fig. 2. Intraoperative photos. (A) After excision of the mass, the cortical bone was relatively intact, however, erosion of the outer table of the skull was observed. (B) The mass of left frontal lesion was well delineated and reddish-brown in color and shows hemorrhagic features with a cluster of small vessels. 
brown, and shows hemorrhagic features with multiple vascular channels (Fig. 2B).

\section{Additional radiologic imaging}

Postoperative computed tomography (CT) scans were performed due to concern about the underlying bone lesions and revealed a mild expansile nature of the mass that expanded in the diploic space in the left frontal bone. In the underlying calvarium of the parietal bone, there was multifocal partial thickness erosion presenting as a honeycomb-shaped geographic osteolytic lesion without neuroparenchymal involvement or discontinuity of the cortical surface on the outer table of the skull. A postoperative CT scan showed the presence of a multifocal intraosseous hemangioma of the parietal bone that did not connected the surgical site (Fig. 3A). Three-dimensional volume rendering technique reconstruction imaging further supported this finding (Fig. 3B). We identified a characteristic "scalloping sign" (depression in the outer or inner table of the skull) of the underlying frontal bone, which was the operation site, and identified a multifocal lesion in the parietal bone (not at the site of operation).

\section{Histologic findings}

Histopathologic examination revealed a mixture of fat, loose fibrocollagenous tissue with extended, thin-walled large dilated veins filled with erythrocytes and sinuses lining a single layer of endothelial cells (Fig. 4A). The pathological features were consistent with a diagnosis of previously known cavernous hemangioma (slow-flow vascular malformation). Multiple small bony trabeculae were also revealed (Fig. 4B).
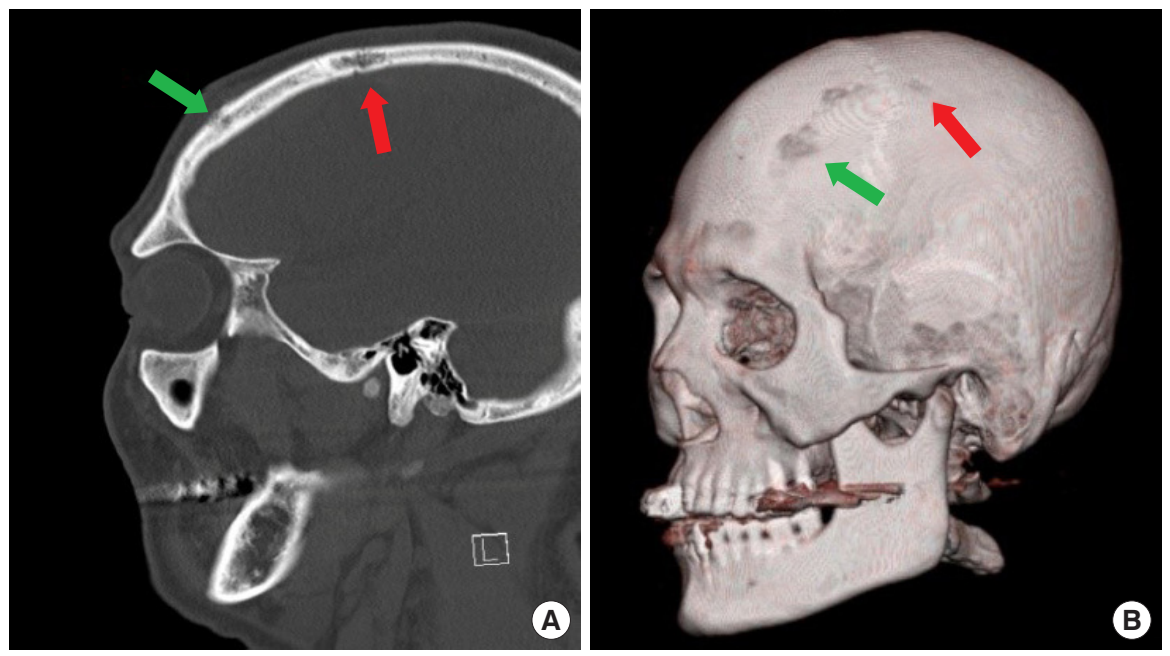

Fig. 3. Computed tomograms. The area where the mass was located in frontal bone (green arrows) and multifocal lesion in parietal bone (red arrows). (A) Sagittal view and (B) three-dimensional volume rendering technique reconstruction image.
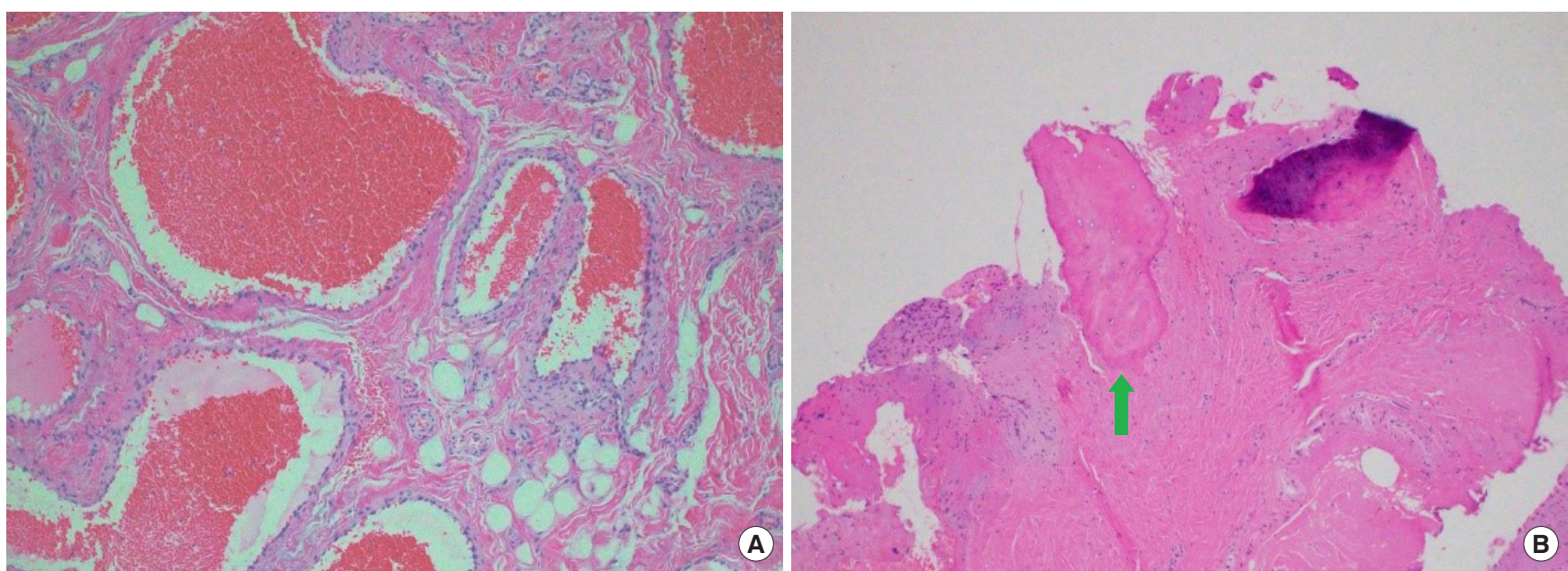

Fig. 4. Histologic findings. (A) The cavernous hemangioma with thin-wall veins of varying sizes (H\&E, $\times 100)$. (B) The cavernous hemangioma with trabecular bone (arrow) $(\mathrm{H} \& \mathrm{E}, \times 10)$. 
We presented the CT findings to the patient and recommended total excision and cranioplasty, however, he refused additional surgery. Instead, we recommended periodic imaging studies and removal of the mass if the size of mass increased or the inner table of calvarium becomes more osteolytic. The postoperative course was uneventful and the contour of the left forehead was well restored.

\section{DISCUSSION}

Primary intraosseous hemangioma is a benign lesion that is typically asymptomatic; occasionally, however, these lesions may appear as a slow-growing hard palpable mass with discomfort, pulsatile sensation or are accidentally detected during image evaluation [5]. The pathogenesis of primary intraosseous hemangioma remains unknown. The causes are considered congenital or traumatic, but this has not yet been proved. Etiology of hemangiomas is not yet known, but trauma history seems to be related in some previously reported cases [3]. Because these tumors tend to expand externally rather than internally, neurologic deficits are uncommon. However, Kanu et al. [6] reported that calvarial hemangioma rarely causes seizure in relation to neurological deficit.

Intraosseous calvarial hemangioma is characteristically recognized in diploic space. They are expansile in nature, widening the diploic space and causing thinning both inner and outer tables of the skull [7]. CT is the most accurate tool for determining the intraosseous range and the involvement of soft tissue (intracranial vs. extracranial location and extent). Characteristic scallops (depression in the outer or inner table of the skull) have been described to distinguish the extracranial and intracranial origins of these lesions. The extracranial lesions produce depression in the outer table of the skull and intracranial lesions produces depression in the inner table of the skull. The CT findings of intraosseous hemangioma may appear as an osteolytic defect with "soap bubble," "honeycomb" or "sunburst" appearances. "Honeycomb" appearances correspond with a scattering of radiodense spots in the radiolucent hollow [6-8]. This radiologic finding is due to osteolytic activity of the mass and secondary reactive osteoblastic remodeling with trabecular bone. Therefore, the differential diagnoses for intraosseous hemangioma include fibrous dysplasia, osteoma, Langerhans cell histiocytosis, and multiple myeloma [3].

Microscopically, multiple engorged vascular elements interspersed among abundant trabecular bones were observed in our patient. The vessels are thin walled and lined with a single endothelial layer [2]. An agglomerate of thin-walled and dilated blood vessels infiltrates the bone, while plump endothelial cells are lined inside the small-caliber thin-walled vessels [1,9].

The treatment of choice for an intraosseous hemangioma is en-bloc resection to the appropriate normal bone margin; the bone defect can be reconstructed in various ways. The others include curettage, radiotherapy, and embolization. The patient in our case did not want to undergo additional treatment, but if the size of the lesion increases on a periodic follow-up, or if the thickness of the inner or outer table of calvarium decreases, total excision of the tumor is recommended.

According to the International Society for the Study of Vascular Anomalies classification system, venous malformation (VM) has been mistakenly called cavernous hemangioma and cavernous angiomas; these terms have led to confusion with the more common proliferating or true hemangioma of infancy for many years. VM is a slow-flow vascular malformation seen at birth, however, it may typically become evident and more prominent as the patient grows up. The most pronounced enlargement is usually seen at the period from infancy to puberty, but less changes may be seen into adulthood [10].

Ultrasonography is the first-line approach to assess soft tissue masses. It can help confirm the presence of a tumor and anatomical location, extent and its relationship to the investing fascia, neurovascular bundle and other tissue without the use of ionizing radiation. Ultrasonography accuracy in diagnosing slow-flow vascular malformations is high when presenting with typical appearance. The sensitivity and specificity of ultrasonography for confirming and diagnosing VM are $73 \%$ and $98 \%$, respectively. However, as in our case, VM is often misdiagnosed as a lipoma when the vascular malformations are of very slow flow and it is composed of mainly echogenic stroma interspersed with small capillaries that are too small to be resolved by ultrasonography [11]. As ultrasonography findings in our case, hypoechoic contents with hyperechoic linear septa can be diagnosed as fibrolipoma, and about $15 \%$ of all lipomas are hypoechoic compare to adjacent fat [12].

We present a rare case of multifocal intraosseous calvarial hemangioma arising in the subgaleal plane causing scalloping of the underlying calvarium in an elderly male patient. Biopsy for intraosseous lesions, of course, is the most accurate, but there was a limitation because the patient did not want to receive any further procedure. In histologic findings of hemangioma including bony trabeculae, we would not be sure whether some of the intraosseous hemangioma is involved in the tissue or whether the subgaleal hemangioma contains part of the cortical bone. However, there were somethings to consider in our case: intraoperative finding also showed that there were multiple vascular channels with bone bleeding, which led us to believe that the subgaleal lesion was connected inward. And the 
outer table of the underlying cortical bone with a hemangioma on CT was very thin. Considering these points, the authors made a diagnosis this would be intraosseous hemangioma. This case highlights the importance of additional CT examination in patients presenting with signs of underlying calvarium scalloping and erosion. Clinicians should also be aware of the possibility of multifocal intraosseous calvarial hemangiomas in lesions that may extend to the periphery and the possibility of intracranial extension, especially in the frontal and parietal bones. Furthermore, proper selection of congenital vascular malformation terminology is still quite confusing and misconception are present in the literature.

\section{NOTES}

\section{Conflict of interest}

No potential conflict of interest relevant to this article was reported.

\section{Ethical approval}

The study was approved by the Institutional Review Board of Chungnam National University Hospital (IRB No. CNUH 2019-02-034) and performed in accordance with the principles of the Declaration of Helsinki. Written informed consent was obtained.

\section{Patient consent}

The patient provided written informed consent for the publication and the use of his images.

\section{ORCID}

Joo-Hak Kim https://orcid.org/0000-0001-9244-0940

Chang Hwan Ahn https://orcid.org/0000-0003-4182-3823

Kyung-Hee Kim https://orcid.org/0000-0003-0214-0296

Sang-Ha Oh https://orcid.org/0000-0003-3734-5005

\section{REFERENCES}

1. Vural M, Acikalin MF, Adapinar B, Atasoy MA. Congenital cavernous hemangioma of the calvaria: case report. J Neurosurg Pediatr 2009;3:41-5.

2. Bastug D, Ortiz O, Schochet SS. Hemangiomas in the calvaria: imaging findings. AJR Am J Roentgenol 1995;164:683-7.

3. Park BH, Hwang E, Kim CH. Primary intraosseous hemangioma in the frontal bone. Arch Plast Surg 2013;40:283-5.

4. Kirmani AR, Sarmast AH, Bhat AR. A unique case of calvarial hemangioma. Surg Neurol Int 2016;7(Suppl 14):S398-401.

5. Kalsi H, Scannell J. Unusual presentation of an intraosseous hemangioma of the maxilla and displaced canine. Int J Clin Pediatr Dent 2013;6:124-6.

6. Kanu OO, Ojo OA, Bankole O, Olaniran A, Anunobi C, Arigbabu SO. Calvarial hemangioma causing seizure disorder: a case report with review of literature. Rom Neurosurg 2012;19: 120-3.

7. Maheshwari S, Arora E, Savant H. Atypical subgaleal haemangioma causing calvarial erosion: a case report. Childs Nerv Syst 2016;32:2475-8.

8. Agrawal A, Jena RK, Reddy U 5th. Scalloping characteristics in a patient with extra-cranial hemangioma. Pol J Radiol 2017; 82:17-8.

9. Khanam H, Lipper MH, Wolff CL, Lopes MB. Calvarial hemangiomas: report of two cases and review of the literature. Surg Neurol 2001;55:63-7.

10. Lee JW, Chung HY. Vascular anomalies of the head and neck: current overview. Arch Craniofac Surg 2018;19:243-7.

11. Hung EH, Griffith JF. Pitfalls in ultrasonography of soft tissue tumors. Semin Musculoskelet Radiol 2014;18:79-85.

12. DiDomenico P, Middleton W. Sonographic evaluation of palpable superficial masses. Radiol Clin North Am 2014;52:1295305. 